\title{
Protective effect of agmatine in acute chlorpromazine hepatotoxicity in rats
}

\author{
Bratislav Dejanovic ${ }^{1}$, Ivana Stevanovic ${ }^{2}$, Milica Ninkovic ${ }^{2}$, Ivana Stojanovic ${ }^{3}$, \\ Vesna Vukovic-Dejanovic ${ }^{4}$ \\ ${ }^{1}$ Military Medical Center Karaburma, Belgrade, Serbia \\ ${ }^{2}$ Institute for Medical Research, Military Medical Academy, Belgrade, Serbia \\ ${ }^{3}$ University of Nis, Faculty of Medicine, Institute for Biochemistry, Nis, Serbia \\ ${ }^{4}$ Insitute for Rehabilitation, Belgrade, Serbia \\ Received March 7, 2014 \\ Accepted September 17, 2014
}

\begin{abstract}
The present study focused on potentially beneficial effects of agmatine on oxidative stress development in the liver during chlorpromazine treatment in rats. We wanted to examine the role of reactive oxygen species and efficiency of antioxidant protection through the determination of malondylaldehyde and total glutathione concentrations in rat liver homogenate, as well as plasma concentrations of malonylaldehyde and sulfhydryl groups after the treatment. Also, liver tissue sections were examined to follow histological changes. Chlorpromazine was applied intraperitoneally at a single dose of $38.7 \mathrm{mg} / \mathrm{kg} \mathrm{b.w}$. The second group was treated with both chlorpromazine (at a single dose of $38.7 \mathrm{mg} / \mathrm{kg} \mathrm{b.w.)} \mathrm{and} \mathrm{agmatine} \mathrm{(at} \mathrm{a} \mathrm{single} \mathrm{dose} \mathrm{of} 75 \mathrm{mg} / \mathrm{kg}$ b.w.). Agmatine was applied immediately after the chlorpromazine. The control group was treated with $0.9 \%$ saline solution in the same manner. Rats were sacrificed by decapitation $24 \mathrm{~h}$ after the treatment and biochemical and immunohistochemical examinations were performed. Analysis of data showed that treatment with agmatine significantly attenuated the oxidative stress indicators as evidenced by lowering malonylaldehyde concentrations in the liver and in plasma while not affecting liver concentrations of total glutathione and plasma concentration of sulfhydryl groups. Additionally, histological evaluation revealed the improvement of liver damage in this respect. The presented data indicated that intraperitoneally administered agmatine protects against chlorpromazine-induced liver disease in rats.
\end{abstract}

Malondialdehyde, glutathione, liver, oxidative stress, plasma

Chlorpromazine (CPZ) is an important member of the phenothiazines, a widely used class of antipsychotic agents. Chronic treatment with neuroleptics increases free radical production (Balijepalli et al. 2001). Oxidative stress and products of lipid peroxidation (LPO) are implicated in the pathophysiology of various neurological disorders. Generation of reactive oxygen species (ROS), such as hydrogen peroxide $\left(\mathrm{H}_{2} \mathrm{O}_{2}\right)$, superoxide radicals $\left(\mathrm{O}_{2}^{-}\right)$, hydroxyl radicals and lipid peroxides are known to damage various cellular components, including membrane lipids, protein, and the DNA, and thereby contribute to cellular dysfunction (Mahadik and Mukherjee 1996). Typical antipsychotics have been shown to increase oxidative stress by altering the concentrations of antioxidant enzymes, and causing oxidative injury, particularly LPO, in the brain (Parikh et al. 2003).

The control of ROS and reactive nitrogen species (RNS) and their cytotoxic bioproducts in affected cells, through scavenging enzymes or thiol repair enzymes, play the main role in tissue repair, protection, and survival. Those antioxidant defense mechanisms include enzymes that scavenge ROS and RNS (catalase, glutathione peroxidase, superoxide dismutase), but also nonenzymatic biomolecules (glutathione-GSH, vitamins $\mathrm{C}$ and $\mathrm{E}$, uric acid).

Chlorpromazine produces a dose-related impairment in bile secretion and altering hepatocyte and canalicular membrane fluidity, which consequently affect the functional

Address for correspondence:

Bratislav Dejanovic, PH, MA

Military Medical Center Karaburma

Severni bulevar 1; Belgrade, Serbia

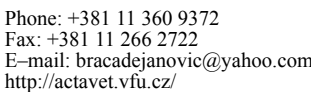

Phone: +381113609372

Fax: +381 112662722

E-mail: bracadejanovic@yahoo.com

http://actavet.vfu.cz/ 
integrity of these sites. Two-week treatment with CPZ orally, leads to inflammatory cells infiltration and focal necrosis (Sulaiman et al. 2006).

Mechanisms responsible for CPZ-induced damage include injury initiated by the activation of Kupffer cells that have been mentioned as mechanisms of toxic CPZ effects. Activated Kupffer cells release proinflammatory cytokines and chemokines which stimulate the migration and accumulation of neutrophils and monocytes in the liver. Activated inflammatory cells amplify CPZ-induced primary injury. Additionally, it has been suggested that hepatocyte injury may be also caused by ischaemia due to sinusoidal endothelial cell dysfunction (Tukov et al. 2007).

The present study was directed to potentially benefit the influence of (4-aminobutyl) guanidine, agmatine (AGM) which is the decarboxylation product of arginine, on oxidative stress development during $\mathrm{CPZ}$ toxicity.

\section{Materials and Methods}

Animals

The experimental animals were treated according to the Guidelines for Animal Study, No. 282-12/2002 (Ethics Committee of the Military Medical Academy, Belgrade, Serbia and Montenegro). Adult male Wistar rats, 2 months old, with body mass $200 \pm 50 \mathrm{~g}$, were used for the experiments. Groups of two or three rats per cage (Erath, FRG) were housed in an air conditioned room at a temperature of $23 \pm 2{ }^{\circ} \mathrm{C}$ with $55 \pm 10 \%$ humidity and with lights on $12 \mathrm{~h} /$ day $(07.00-19.00 \mathrm{~h})$. The animals were given a commercial rat diet and tap water ad libitum.

\section{Experimental procedure}

The animals were divided into 4 groups and treated as follows: 20 rats received normal saline intraperitoneally (i.p.), serving as sham control group (CG group); 20 animals were treated with a single daily dose $(38.7 \mathrm{mg} / \mathrm{kg})$ i.p. of CPZ-HCl (Medisca, Milan-Italy) followed by normal saline i.p. (CPZ group); 20 animals were treated with $38.7 \mathrm{mg} / \mathrm{kg} \mathrm{CPZ}-\mathrm{HCl}$ i.p. followed by treatment with $75 \mathrm{mg} / \mathrm{kg}$ daily doses i.p. of AGM sulfate (Sigma Aldrich, Germany) (CPZ+AGM group); and 20 animals treated i.p. with normal saline followed by a single daily dose $(75 \mathrm{mg} / \mathrm{kg}$ ) of AGM i.p. (AGM group). The total number of animals in the study was 80 . The rats were sacrificed by decapitation $24 \mathrm{~h}$ after the respective treatment. Blood samples for determining oxidative stress indicators were collected from vena iliaca externa. For the same purpose livers were excised and stored at $-20^{\circ} \mathrm{C}$.

Tissue preparation

The livers from five animals from each of the experimental groups were dissected and rapidly placed in $30 \%$ sucrose for at least 3 days. The livers were frozen in methylbutane and stored at $-70{ }^{\circ} \mathrm{C}$ until cryosectioning (CRIOCUT-E Reichert-Yung). Livers from the remaining animals $(\mathrm{n}=15)$ were washed in $0.9 \%$ saline solution, homogenated on ice with Teflon ${ }^{\circledR}$ pounder and stored at $-20{ }^{\circ} \mathrm{C}$ for later biochemical analysis.

\section{Biochemical analyses}

The tissue for biochemical analysis was prepared by the method of Gurd et al. (1974). Total protein concentration was estimated with bovine serum albumin as a standard (Lowry et al. 1951).

Lipid peroxidation analysis in the liver homogenates and in the plasma were measured as malondialdehyde (MDA) production, assayed in the thiobarbituric acid reaction as described by Girotti et al. (1991). The results are expressed as $\mathrm{nmol} / \mathrm{mg}$ proteins in liver homogenates or $\mu \mathrm{mol} / \mathrm{l}$ in $\mathrm{plasma}$.

Total liver glutathione content was determined with DTNB-GSSG reductase recycling assay. The rate of formation of 5-thio-2-nitrobenzoic acid (TNB), which is proportional to the total GSH concentration, was followed spectrophotometrically at $412 \mathrm{~nm}$ (Anderson 1986). The results are expressed as $\mathrm{nmol} \mathrm{of} \mathrm{GSH} / \mathrm{mg}$ proteins.

Plasma concentration of total sulfhydryl $(\mathrm{SH})$ groups was measured spectrophotometrically at $412 \mathrm{~nm}$ in phosphate buffer ( $0.2 \mathrm{~mol}+2 \mathrm{mmol}$ EDTA, pH 9) using 5,5-dithiobis-2-nitrobenzoic acid (DTNB, $0.01 \mathrm{M})$ (Elman 1959). The results are expressed as $\mu \mathrm{mol}$ of $\mathrm{SH} / \mathrm{l}$.

Immunohistochemistry

Frozen, $8 \mathrm{~mm}$ thick liver sections were deposited on poly-L-lysine coated slides and left to air dry. Immunoperoxidase staining was performed by an EnVision antibody complex method using the DakoCytomation EnVision + System-HRP kit (Dako, Glostrup, Denmark). We used the following primary antibodies: a mouse monoclonal ED1, anti-CD68 antibody that recognizes a lysosomal membrane-related antigen on macrophages/ microglia (dilution 1:10, Abcam, Cambridge, UK) and a mouse monoclonal OX42, anti CD11b/c equivalent antibody that reacts with a common epitope shared by $\mathrm{CD} 11 \mathrm{~b} / \mathrm{c}$ which is expressed on monocytes/macrophages and microglia (dilution 1:100, Abcam, Cambridge, UK). 
Reagents

All chemicals used in this study were of analytical grade and from the mentioned sources. All drug solutions were prepared on the day of the experiment.

Statistical analysis

After verifying a normal distribution in all groups using the Kolmogorov-Smirnov test, the data were presented as mean \pm SEM. Immunohistochemical and biochemical data were analyzed statistically by One Way ANOVA using Dunnett's $\mathrm{C}$ test. The linear regression analysis was performed to determine the relation between immunohistochemical and biochemical indicators, using the statistical program GraphPad Prism. Statistical significance was defined as $P<0.05$.

\section{Results}

The results of our study found that CPZ treatment induced significant changes in the indicators of oxidative stress and antioxidant capacity in liver samples and plasma of experimental animals. The treatment of rats with AGM $(75 \mathrm{mg} / \mathrm{kg}$ b.w. i.p. $)$ alone did not significantly affect any of the measured indices compared to rats that received only saline (Figs 1A,B, 2A,B).

A significant rise in liver MDA concentration was found $24 \mathrm{~h}$ after administration of CPZ i.p. $(P<0.05)$ (Fig. 1A). Similar to this, plasma MDA concentration was significantly higher in the CPZ-treated group in comparison with the control group at the same time interval (Fig. 1B). Our results show hepatoprotective effects of AGM, which can reduce the concentration of MDA in the liver and plasma (Fig. 1A,B). There is a significant negative correlation (Fig. 1C) between MDA concentration in the liver and plasma of CPZ-treated animals $(\mathrm{r}=-0.89, P<0.0001)$.

Liver GSH concentration showed a tendency to rise in the CPZ+AGM-treated group compared to the CPZ group $(P<0.05)$ (Fig. 2A). Here, the obtained results also suggest a significant negative correlation (Fig. $2 \mathrm{C}$ ) between $\mathrm{SH}$ concentrations in plasma with $\mathrm{OX} 42$ expression in CPZ-treated animals $(\mathrm{r}=-0.93, P<0.0001)$.

The number and density of ED1 (Plate IV, Fig. 3A, sections A-D) and OX42 (Fig. 3B, sections A-D) positive cells (monocytes and macrophages) was markedly increased in CPZ group $(+++)$, than in AGM $(+)$ or CPZ+AGM samples $(++)(P<0.05)$ (Table 1$)$. No labelling of cells for ED1 (Fig. 3A, section A) and OX42 (Fig. 3B, section A) were seen $24 \mathrm{~h}$ after saline solution injection in rats. The obtained results suggest the significant positive correlation (Fig. 3C) between ED1 expression with OX42 expression in CPZtreated animals $(\mathrm{r}=0.84, P<0.005)$.

Table 1. Summary of immunohistochemical examinations of rats liver sections.

\begin{tabular}{lcc}
\hline Group & \multicolumn{3}{c}{ Antibody } & \\
\cline { 2 - 3 } & ED1 & OX42 \\
\hline CG & - & - \\
CPZ & +++ & +++ \\
CPZ+AGM & ++ & ++ \\
AGM & + & + \\
\hline
\end{tabular}

CG-control group; CPZ-chlorpromazine; AGM-agmatine. (-) absence of labelled cells; $(+)$ weak reaction; $(++)$ intermediate reaction; $(+++)$ strong reaction.

\section{Discussion}

The results of our investigation have shown the important role of oxidative stress in acute CPZ-induced liver injury. Our study has shown that liver and plasma MDA concentrations were significantly increased in the $\mathrm{CPZ}$ treated group in comparison with the control group 


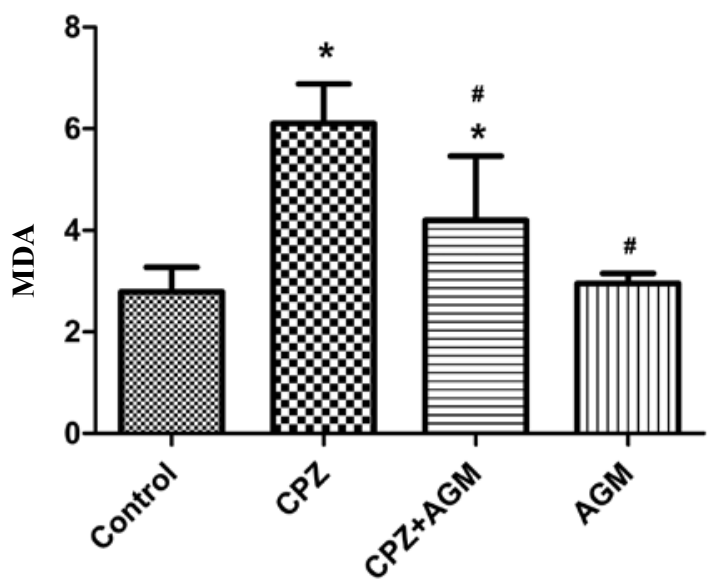

Fig. 1A. Malondialdehyde concentration (nmol/mg protein) in rat liver tissue.

C-control; CPZ-chlorpromazine; AGM-agmatine. The data are expressed as mean $\pm \mathrm{SEM}$. * - compared to control group; \# - compared to CPZ group. Significance was considered at: $P<0.05$, One Way ANOVA, Dunnett's C test.

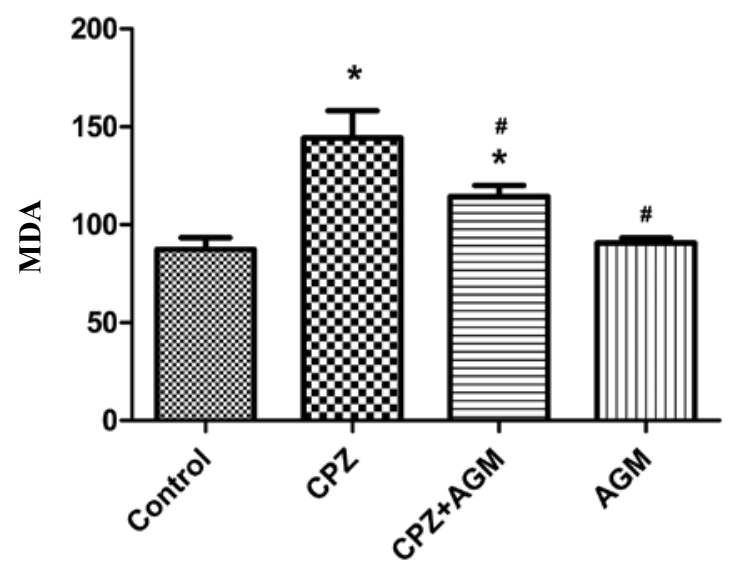

Fig. 1B. Malondialdehyde concentration $(\mu \mathrm{mol} / \mathrm{l})$ in rat plasma.

$\mathrm{C}$-control; CPZ-chlorpromazine; AGM-agmatine. The data are expressed as mean $\pm \mathrm{SEM}$. * - compared to control group; \# - compared to CPZ group. Significance was considered at: $P<0.05$, One Way ANOVA, Dunnett's C test.

$24 \mathrm{~h}$ after its administration (Fig. 1A, B). It is known that LPO is an important mechanism in acute and chronic CPZ intoxication (Khatua and Bhattacharyya 2001). Previous studies have indicated the implication of oxidative stress in hepatic tissue damage induced by CPZ treatment, manifested by MDA elevation in liver tissue (Parola et al. 1996), which can be explained as a consequence of generation of $\mathrm{CPZ}$ cation radicals and/or metabolic activation of CPZ to quinoneimine derivatives (Toler 2004). Retention of hydrophobic bile acids and toxic substances and infiltration of inflammatory cells may participate in the generation of ROS with consequent production of oxidative damage (Casini et al. 


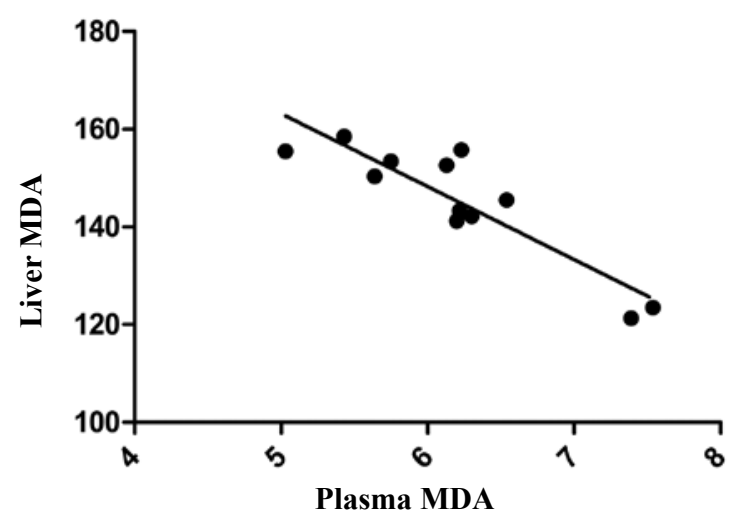

Fig. 1C. The correlation of malondialdehyde concentration (nmol/mg protein) in liver homogenates and malondialdehyde concentration $(\mu \mathrm{mol} / 1)$ in plasma of CPZ-treated animals. Significance was considered at: $P<$ 0.0001 , Pearson's correlation, $r=-0.89$.

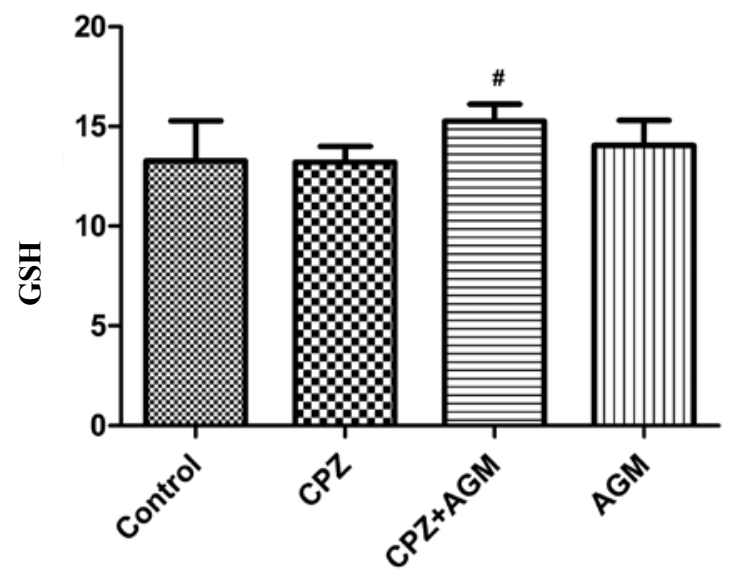

Fig. 2A. Glutathione concentration (nmol/mg protein) in rat liver tissue.

C-control; CPZ-chlorpromazine; AGM-agmatine. The data are expressed as mean $\pm \mathrm{SEM}$. \# - compared to CPZ group. Significance was considered at: $P<0.05$, One Way ANOVA, Dunnett's $C$ test.

1997). The results of this study indicate that MDA concentrations in rat liver homogenate were significantly negatively correlated with plasma concentrations of MDA $(P<0.0001$, $\mathrm{r}=-0.89$ ) $24 \mathrm{~h}$ after CPZ administration (Fig. 1C).

It is also known that AGM has hepatoprotective effects that may be related to its ability to suppress oxidative stress, NO synthesis and TNF- $\alpha$ production (El-Agamy et al. 2014; Rushaidhi et al. 2013). The beneficial effect of AGM could relate to the ability of AGM to inhibit iNOS or to block NMDA receptors and/or voltage-dependent $\mathrm{Ca}^{2+}$ channels (Yang and Reis 1999). Blockade of either of these signalling proteins would likely be protective. Our results showed a reduction in both the concentration of MDA in the liver (Fig. 1A) and in plasma (Fig. 1B) after the administration of AGM. Plasma concentrations of MDA were 


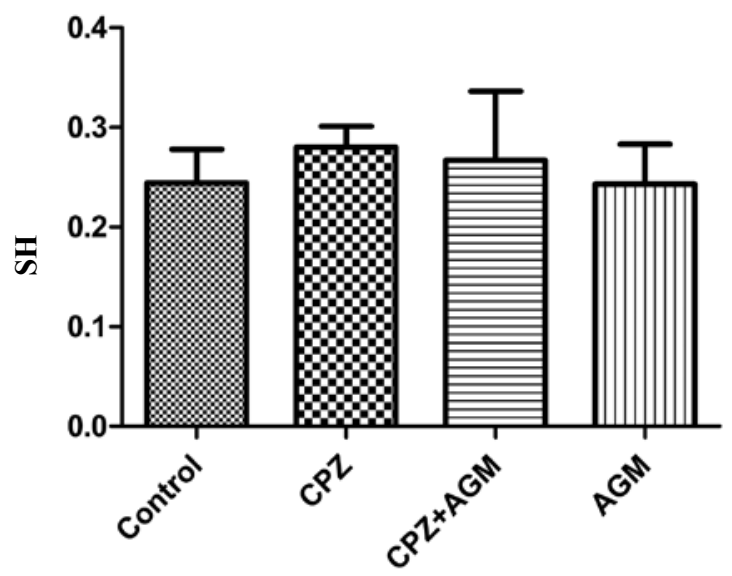

Fig. 2B. Concentration of sulfhydryl groups ( $\mu \mathrm{mol} / \mathrm{mg}$ protein) in rat plasma.

C-control; CPZ-chlorpromazine; AGM-agmatine. No significant difference was found between experimental groups.

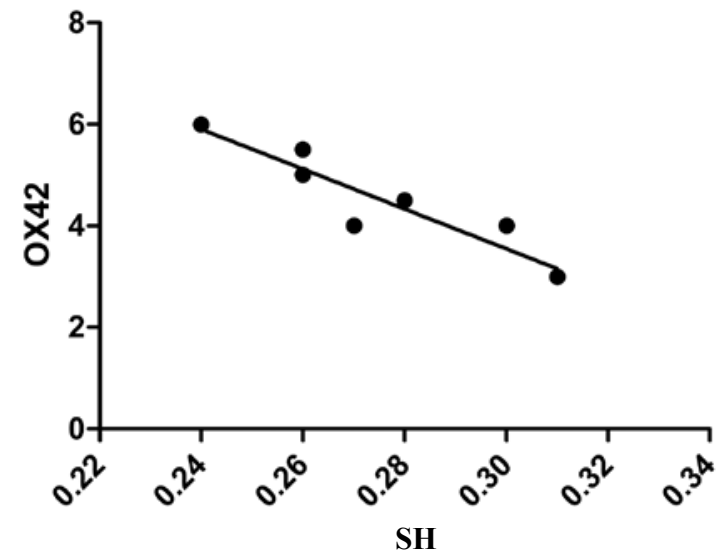

Fig. 2C. The correlation of sulfhydryl concentration ( $\mu \mathrm{mol} / \mathrm{mg}$ protein) in plasma with OX42 expresssion of CPZtreated animals. Significance was considered at: $P<0.0001$, Pearson's correlation, $\mathrm{r}=-0.93$.

significantly negatively correlated with ED1 expression $(P=0.0187, \mathrm{r}=-0.94)$ and $\mathrm{OX} 42$ expression $(P=0.0054, \mathrm{r}=-0.97)$ in CPZ+AGM group (data not shown).

The found unchanged concentrations of GSH in acute CPZ intoxication suggest that GSH depletion is not the primary mechanism of oxidative stress in acute CPZ liver injury (Fig. 2A). Absence of change of liver GSH content can be explained by simultaneous activation of adaptive mechanisms in hepatocytes. Similar to GSH, plasma concentration of SH groups was not significantly changed within the first $24 \mathrm{~h}$ after $\mathrm{CPZ}$ administration (Fig. 2B). Since GSH is the most abundant source of SH groups in the liver, changes in $\mathrm{SH}$ groups concentration (Fig. 2B) are in accordance with the changes in liver GSH content (Fig. 2A). This study also found a negative linear relationship between decreased plasma 


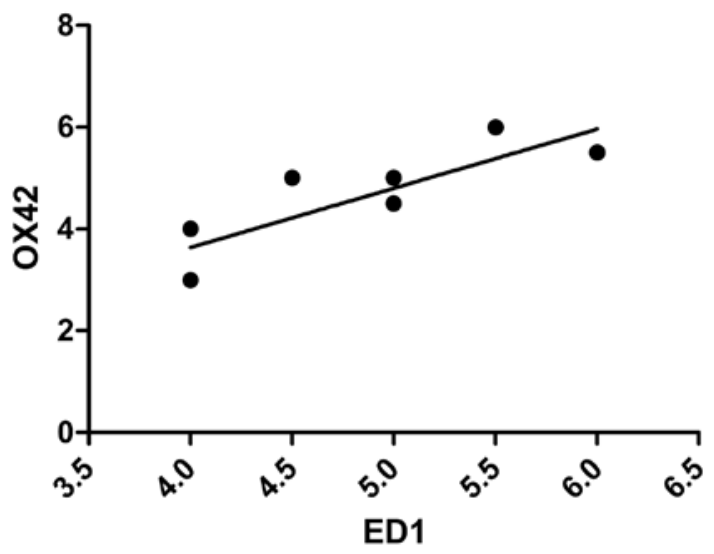

Fig. 3C. The correlation of ED1 antibody expression and OX42 antibody expression in CPZ-treated animals. Significance was considered at: $P<0.005$, Pearson's correlation, $\mathrm{r}=0.84$.

concentration of SH groups and increased OX42 expression in liver samples (Fig. 2C) of CPZ-treated animals $(P<0.0001, \mathrm{r}=-0.93)$. This effect of negative correlation could be just a result of antioxidative defence damage and macrophage activation.

Under physiological conditions, GSH synthesis in cells is regulated by bioavailability of previously formed GSH. The increase in hepatic GSH concentration after CPZ+AGM administration results from the inhibition of GSH efflux across the canalicular membrane of hepatocyte, since GSH represents the major osmotic solute for the generation of bile salt-independent bile flow. The protective effect of AGM observed in this respect can be attributed to its high efficacy in scavenging various types of free radicals, and enhancement of antioxidant enzymes activities (Bhutada et al. 2012).

Studies have shown that CPZ causes morphological changes of the treated rats; the mainly observed pathological changes included: cholestasis manifested by feathery changes, proliferation of bile duct, appearance of pigmented granules and intracellular vacuoles within hepatocytes, inflammatory cell infiltration, ground glass appearance and hydropic degeneration (Sulaiman et al. 2006). Immunohistochemical examination of both ED1 and OX42 expression in the liver of adult rats was performed to demonstrate the occurrence of activated positive macrophages. ED1 is a pan-macrophage marker and is expressed intracellularly on lysosomes. Under the condition of this experiment in CPZtreated animals (Plate IV, Fig. 3A, section B; $P<0.05$ ), we observed an increase in ED1 expression, resulting in a higher number $(+++)$ of positive cells (Table 1$)$. Also, the results show a massive region-specific increase in macrophages and OX42 expressing cell profiles in aged rats (Plate VI, Fig. 3B, section $\mathrm{B} ; P<0.05$ ). Our data suggest that macrophagepositive cells were present in AGM-treated animals $(+)$ and enhanced in CPZ+AGMtreated animals $(++) 24 \mathrm{~h}$ after drug administration, with maximum expression $24 \mathrm{~h}$ after CPZ application $(+++)$ in the liver (Table 1). ED1 expression was positively associated with OX42 expression $(P=0.0021, \mathrm{r}=0.84)$ in liver samples, implying the importance of macrophage activation in acute CPZ-induced liver injury (Fig. 3C).

Based on our results, it can be concluded that oxidative stress, among various mechanisms, plays an important role in acute CPZ-induced liver injury in rats. The results show that LPO occurs within $24 \mathrm{~h}$ after CPZ administration, and that GSH and SH groups do not have an important protective role in early CPZ intoxication. Since CPZ induces oxidative 
stress by activating ROS that lead to tissue damage, the study pointed to the potentially protective effect of AGM and its ability to correct changes in indicators of oxidative stress induced by CPZ. It can be concluded that AGM reduces liver and plasma concentrations of MDA in CPZ-induced injury which indicate that AGM might have a protective effect against oxidative membrane damage.

\section{Acknowledgement}

This work was supported by a grant from the project: МФВМА/3/13-15 in MФВМA/6/12-14 funded by the Military Medical Academy. No conflict of interest exists for any of the authors of this article.

\section{References}

Anderson ME 1986: The DTNB-GSSG reductase recycling assay for total glutathione (GSH + 1/2GSSG). In: Greenwald RA (Ed.): Tissue Glutathione. Boca Raton, CRC Press, pp. 317-323

Balijepalli S, Kenchappa RS, Boyd MR, Ravindranath V 2001: Protein thiol oxidation by haloperidol results in inhibition of mitochondrial complex I in brain regions: comparison with atypical antipsychotics. Neurochem Int 38: $425-435$

Bhutada P, Mundhada Y, Humane V, Rahigude A, Deshmukh P, Latad S, Jain K 2012: Agmatine, an endogenous ligand of imidazoline receptor protects against memory impairment and biochemical alterations in streptozotocin-induced diabetic rats. Prog Neuropsychopharmacol Biol Psychiatry 37: 96-105

Casini A, Ceni E, Salzano R, Biondi P, Parola M, Galli A, Foschi M, Caligiuri A, Pinzani M, Surrenti C 1997: Neutrophil-derived superoxide anion induces lipid peroxidation and stimulates collagen synthesis in human hepatic stellate cells: role of nitric oxide. Hepatology 25: 361-367

El-Agamy DS, Makled MN, Gamil NM 2014: Protective effects of agmatine against D-galactosamine and lipopolysaccharide-induced fulminant hepatic failure in mice. Inflammopharmacology 22: 187-194

Elman GL 1959: Tissue sulfhydryl groups. Arch Biochem Biophys 82: 70-77

Girotti M, Khan N, Mc Lellan B 1991: Early measurement of systemic lipid peroxidation products in the plasma of major blunt trauma patients. J Trauma 31: 32-35

Gurd JW, Jones LR, Mahler HR, Moore WJ 1974: Isolation and partial characterization of rat brain synaptic membrane. J Neurochem 22: 281-290

Khatua AK, Bhattacharyya M 2001: NADPH-induced oxidative damage of rat liver microsomes: protective role of chlorpromazine and trifluoperazine. Pol J Pharmacol 53: 629-634

Lowry OH, Rosenbrongh NJ, Farr AL, Randal RJ 1951: Protein measurement with the folin phenol reagent. J Biol Chem 193: 265-275

Mahadik SP, Mukherjee S 1996: Free radical pathology and antioxidant defense in schizophrenia: a review. Schizophr Res 19: 1-8

Parikh V, Khan MM, Mahadik SP 2003: Differential effects of antipsychotics on expression of antioxidant enzymes and membrane lipid peroxidation in rat brain. J Psychiatry Res 37: 43-51

Parola M, Leonarduzzi G, Robino G, Albano E, Poli G, Dianzani MU 1996: On the role of lipid peroxidation in the pathogenesis of liver damage induced by long-standing cholestasis. Free Radic Biol Med 20: 351-359

Rushaidhi M, Zhang H, Liu P 2013: Effects of prolonged agmatine treatment in aged male Sprague-Dawley rats. Neuroscience 234: 116-124

Sulaiman A, Al-Shawi N, Jwaied A, Mahmood D, Hussain S 2006: Protective effect of melatonin against chlorpromazine-induced liver disease in rats. Saudi Med J 27: 1477-1482

Toler SM 2004: Oxidative stress plays an important role in the pathogenesis of drug-induced retinopathy. Exp Biol Med 229: 607-615

Tukov FF, Luyendyk JP, Ganey PE, Roth RA 2007: The role of tumor necrosis factor alpha in lipopolysaccharide/ ranitidine-induced inflammatory liver injury. Toxicol Sci 100: 267-280

Yang XC, Reis DJ 1999: Agmatine selectively blocks the N-methyl-D-aspartate subclass of glutamate receptor channels in rat hippocampal neurons. J Pharmacol Exp Ther 288: 544-549 
Plate IV

Dejanovic B. et al.: Protective effect ... pp. 305-312
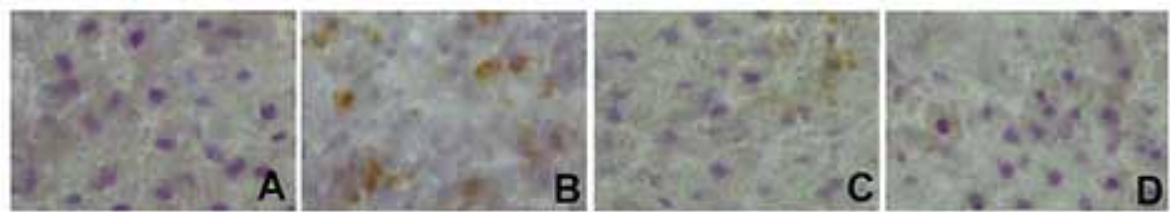

Fig. 3A. The representative photomicrographs of ED1 staining by immunohistochemistry of liver sections in control group (A), CPZ group (B), CPZ+AGM group (C) and AGM group (D). The number of tested rats was five for each group. Original magnification $\times 400$. The data were statistically compared between groups by Student's $t$-test $(P<0.05)$.
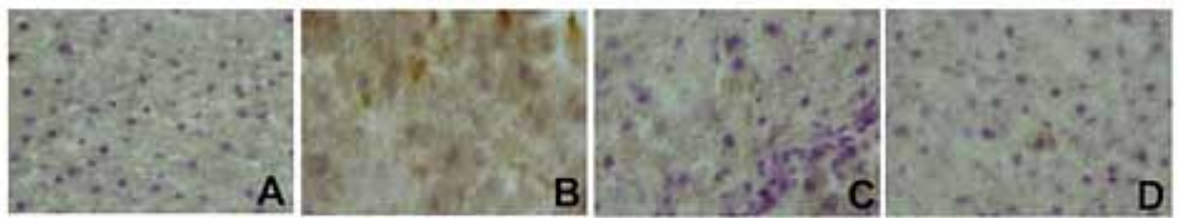

Fig. 3B. The representative photomicrographs of OX42 staining by immunohistochemistry of liver sections in control group (A), CPZ group (B), CPZ+AGM group (C), and AGM group (D). The number of tested rats was five for each group. Original magnification $\times 400$. The data were statistically compared between groups by Student's $t$-test $(P<0.05)$. 